\title{
LISA Observations of Supermassive Black Hole Growth
}

\author{
Miroslav Micic, Kelly Holley-Bockelmann and Steinn Sigurdsson \\ Pennsylvania State University, 525 Davey Lab, University Park, 16802
}

\begin{abstract}
Based on a high resolution cosmological n-body simulation, we track the hierarchical growth of black holes in galaxy clusters from $z=20$ to $z=0$. We present a census of black holes as function of redshift and will determine their mass assembly history under a variety of assumptions regarding the importance of gas accretion in black hole growth, from early supercritical Eddington accretion to gas-poor hierarchical assembly. Following a galaxy merger, black holes are expected to form, inspiral and merge after strongly radiating energy via gravitational waves. For each binary black hole inspiral and merger, we determine the expected gravitational wave signal for the Laser Interferometer Space Antenna (LISA), and calculate the LISA event rate as a function of time. We will calculate the black hole mass assembly history for several black hole growth scenerios, so that we can explore tests to characterize each model observationally. In particular, we will study how well LISA observations will be able to distinguish between these very different assembly scenarios.
\end{abstract}

Keywords: Pop III, IMBH, SMBH, DMH, gravitational radiation, LISA, BBO

PACS: $98.65 \mathrm{Fz}$

\section{MOTIVATION}

In current theories of structure formation, the first generation of star formation seeds intermediate mass black holes deep within the potential of forming galaxies (Madau \& Rees 2001, Abel et al. 2002, Heger et al. 2003). Mass of the structures that can host first massive black holes is limited by various feedback processes. From Population III supernova studies, the formation of first stars stops at $\mathrm{z} \sim 12$ (Wise \& Abel 2005). These black holes can grow in mass by accreting gas, and as the event horizon grows in size, stars and stellar remnants. Later, galaxy mergers provide an impetus for these growing black holes to meet and form a bound system (Volonteri et al. 2003, Islam et al. 2003). During a galaxy merger, each black hole sinks to the center of the new galaxy potential due to dynamical friction, and eventually becomes bound as a binary. Dynamical friction then continues to shrink the orbit until the binary is hard (i.e., the separation between each black hole, $a_{\mathrm{BBH}}$, is such that the system tends to lose energy during stellar encounters)(Madau \& Rees 2001, Holley-Bockelmann et al. 2001, Vine $\&$ Sigurdsson 1998). Thereafter, further decay is mediated by 3-body scattering with the ambient stellar background until the binary becomes so close that the orbit can lose energy via gravitational radiation. In studies of a static, spherical potential, it may be difficult for stellar encounters alone to cause the binary to transition between the 3body scattering phase and the gravitational radiation regime; however in gas-rich or non-axisymmetric systems the binary hardens efficiently into one that emits copious gravitational radiation. Thereafter, it presumably coalesces. Depending on the black hole mass, the final stages of coalescence emit so much gravitational radiation (Thorne 

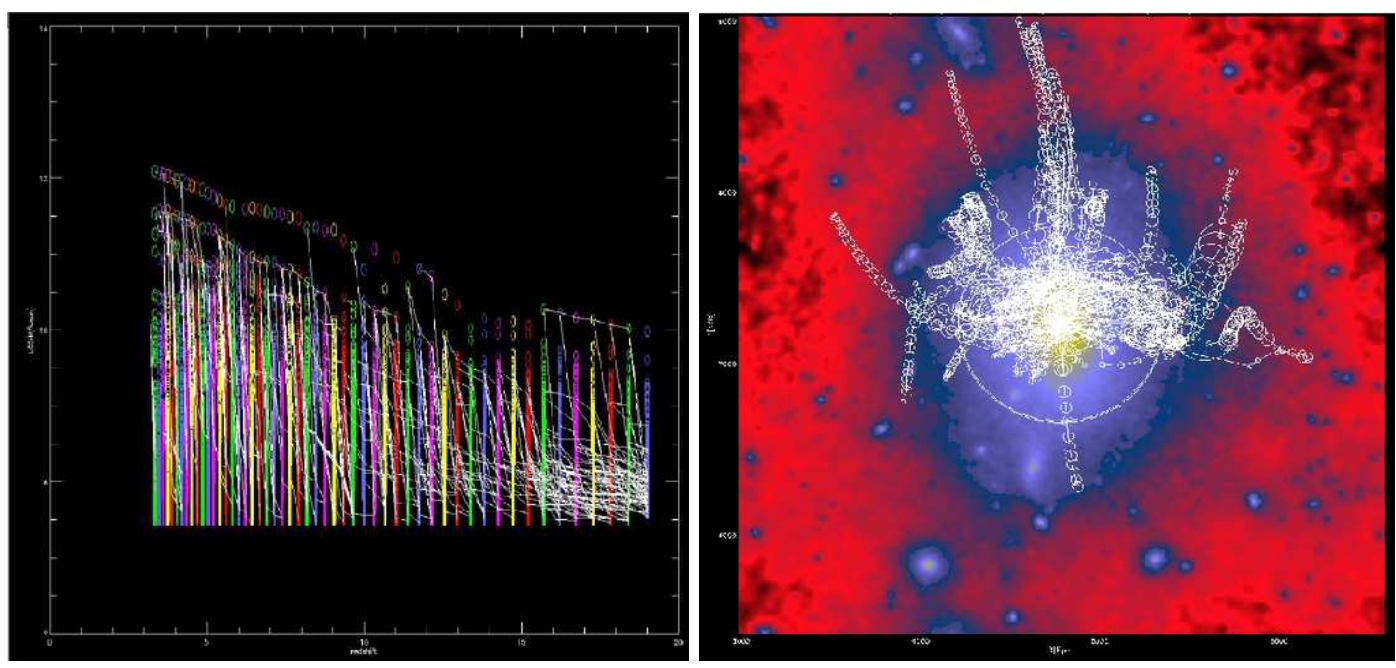

FIGURE 1. 1a-left- Merger tree from $z=20$ to $z=3$. Different halos are represented with different colors. The sizes of the circles are set by the virial radius, which is related to $M_{\text {halo,virial }}^{1 / 3}$. By our dry merger assumption, $M_{\mathrm{BH} 1}$, is also related to $M_{\text {halo,virial }}$. Given this merger tree, we will test different models for black hole accretion. 1b-right- 2D density projection of the simulation box with primary halo. Overploted are paths of dark matter halos which grow in mass (circles represent scaled virial radii) and sink toward primary. Only main branches of the merger tree are presented.

1995, Cornish \& Levin 2002, Vecchio et al. 2004) that they are extremely likely to be detected by the Laser Interferometer Space Antenna (LISA), a planned NASA mission to detect gravitational waves, set to launch in the next decade. Current estimates of the total gravitational wave signal from the cosmological growth and merger lifecycle)of black holes have been made using semianalytic models of merger trees, with analytic prescriptions for the black hole merger timescales within the analytic halos and models for gas accretion. In this study, we use high resolution N-body simulations to track the seed black holes themselves as they sink within a galaxy halo. We will then use this to more accurately model different black hole growth scenerios, which will yield different gravitational wave signatures. Ultimately, we will determine if LISA observations can be used as a tool to discover how black holes grow.

\section{GROWING SUPERMASSIVE BLACK HOLES}

In our numerical simulations we use GADGET to evolve a comoving $14.3 \mathrm{Mpc}^{3}$ section of a $\Lambda$ CDM universe $\left(\Omega_{M}=0.3, \Omega_{\Lambda}=0.7\right.$ and $\left.\mathrm{h}=0.7\right)$ from $z=40$ to $z=0$. We refine a sphere of $2 \mathrm{Mpc}$ in the box to simulate at higher resolution with $4.9 \times 10^{6}$ high-resolution particles (softening length $2 \mathrm{kpc}$ comoving). The rest of the box has $2.0 \times 10^{6}$ lowresolution particles (softening length $4 \mathrm{kpc}$ comoving). The mass of each high resolution particle in this simulation is $8.85 \times 10^{5} \mathrm{M}_{\odot}$ and the mass of each low-resolution particle is $5.66 \times 10^{7} \mathrm{M}_{\odot}$. We use P-GroupFinder to define dark matter halos and to identify black holes as the most bounded particles in their host halos.

Only dark matter halos with mass $\mathrm{M}_{v i r} \geq 10^{8} \mathrm{M}_{\odot}[(1+z) / 10]^{-1.5}$ can host first stars and 

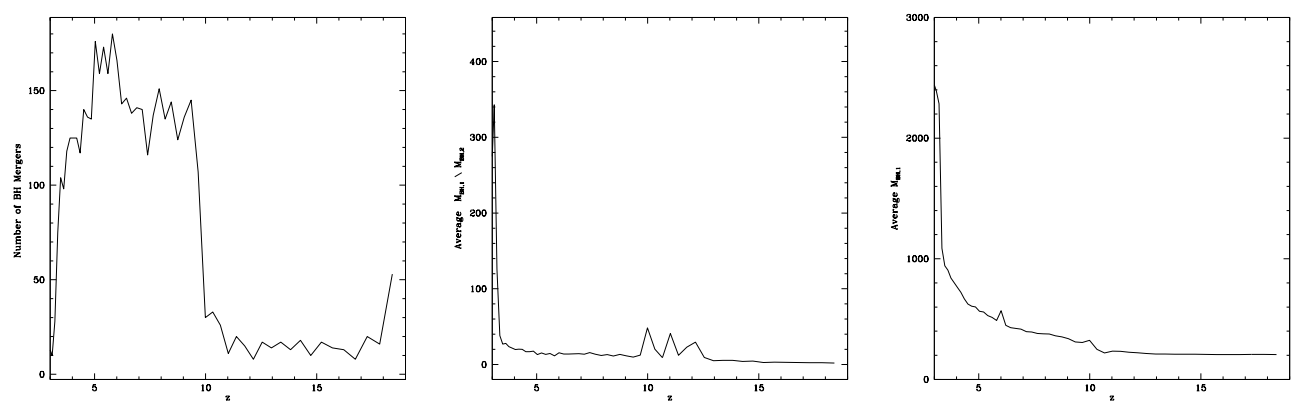

FIGURE 2. 2a-Left- Number of IMBHs mergers as a function of redshift. 2b-Middle- Average ratio of merging black holes as a function of redshift. 2c-Right- Average of more massive components in the merger as a function of redshift.

the first stars stop forming at $\mathrm{z} \sim 12$. By tracking the positions of over 3800 seed black holes and their host halos throughout the simulation (Fig. 1), we will model different black hole merger scenerios and track the growth of the black hole mass per halo as a function of time and environment. In particular, we will parameterize the degree of gas accretion involved with growing the black hole and the efficiency of binary coalescence.

In this first attempt, however, we model the seed black hole merger timescale and the mass accretion history in the simplest possible way. We assume that the hardening timescale is rapid, that no black holes are ejected, and that there is no gas accretion involved. This gives us a fiducial model with which to compare more realistic black hole growth scenerios.

\section{DRY MERGER AND MERGERS PLUS ACCRETION}

We start with $200 \mathrm{M}_{\odot}$ initial black hole mass. Given this simple growth scenerio, we find a large number of mergers at each redshift, with the largest number of mergers at $z \sim 6$. At later times, 100: 1 mergers are much more common, as $M_{\mathrm{BH} 1}$ grows larger while also accreting smaller mass black holes from satellite halos (Fig. 2). At this stage we have analyzed the data in range $3 \leq z \leq 20$. The intensity of mergers of dark matter structures demands more detailed analysis, to be addressed in the future work. Notice that dry mergers build massive black holes $\mathrm{M} \geq 10^{6} \mathrm{M}_{\odot}$ by redshift $\mathrm{z}=3$. We add accretion to the previously described growth of massive black holes through mergers. For a sustained Eddington accretion of baryon matter the mass growth rate is $\mathrm{M}_{B H} / \mathrm{t}_{S a l}$, where $\mathrm{t}_{S a l}$ is Salpeter time-scale, $\mathrm{t}_{\mathrm{Sal}} \sim 4 \times 10^{7} \mathrm{yr}$ from the recent observations. Accretion is triggered by mergers of dark matter halos and lasts $t_{S a l}$. During this time black holes doubles its mass.

\section{EXTRACTING GRAVITATIONAL WAVES}

We assume the black hole binary only emits gravitational radiation during the 'gravitational wave' phase, where the orbital separation of the binary decays most rapidly due 

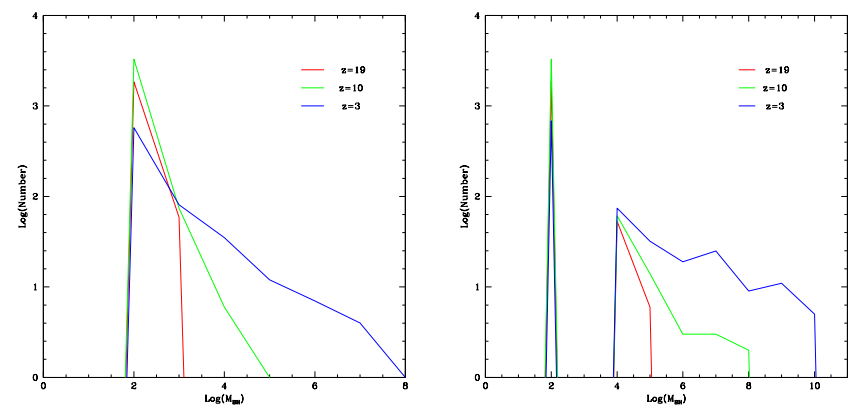

FIGURE 3. 3a-left- Mass function for merging black holes. Red - redshift $\mathrm{z}=19$; Green - redshift $\mathrm{z}=10$; Blue - redshift $z=3$. 3b-right- Mass function for massive black holes which grew through mergers and accretion. There is a number of supermassive black holes at redshift $\mathrm{z}=3$.

to gravitational wave emission. In reality, this is a lower limit to the amount of gravitational radiation emitted during a typical binary's evolution because gravitational radiation is also produced during the hardening phase, as well as when the binary coalesces and rings down.

Under this assumption, the maximum rest frame frequency $f_{r}$, occurs for a circular orbit at 3 Schwarzschild Radii:

$$
f_{\max }=\frac{c^{3}}{14.7 \pi G} \frac{\left(M_{1}+M_{2}\right)^{1 / 2}}{M_{1}^{3 / 2}}
$$

where $\mathrm{M}_{1}$, and $\mathrm{M}_{2}$ are the black hole masses, $\mathrm{G}$ is the gravitational constant, and $\mathrm{c}$ is the speed of light. The minimum $f_{r}$ is:

$$
f_{\min }=\frac{1}{\pi} \frac{G\left(M_{1}+M_{2}\right)^{1 / 2}}{a_{g w}^{3}}
$$
by:

where $\mathrm{a}_{g_{w}}$ is the binary separation where gravitational radiation dominates, expressed

$$
a_{g w}=0.0014 p c\left[\frac{M_{1} M_{2}\left(M_{1}+M_{2}\right)}{10^{18.3} M_{\odot}^{3}}\right]^{1 / 4} t_{g}{ }^{1 / 4}
$$

where $\mathrm{t}_{g}{ }^{1 / 4}$ is the coalescence timescale in Gyr. The change in the rest frame frequency is:

$$
\dot{f}_{r}=\frac{96}{5} f_{r}^{5 / 3} \frac{G^{3} M_{1} M_{2}\left(M_{1}+M_{2}\right)}{c^{5} a^{3}}\left[\frac{G\left(M_{1}+M_{2}\right)}{\pi^{2}}\right]^{-1 / 3}
$$

So, for each merger at a comoving distance $\mathrm{d}(\mathrm{z})$, gravitational radiation is emitted with a characteristic strain, $\mathrm{h}_{c} f_{r}$

$$
h_{c}\left(f_{r}\right)=\frac{g(\varepsilon) 8 \pi^{2 / 3} G^{5 / 3} M_{c h i r p}{ }^{5 / 3} f_{r}^{5 / 3}}{3.16 c^{4} d(z) \dot{f}_{r}}
$$



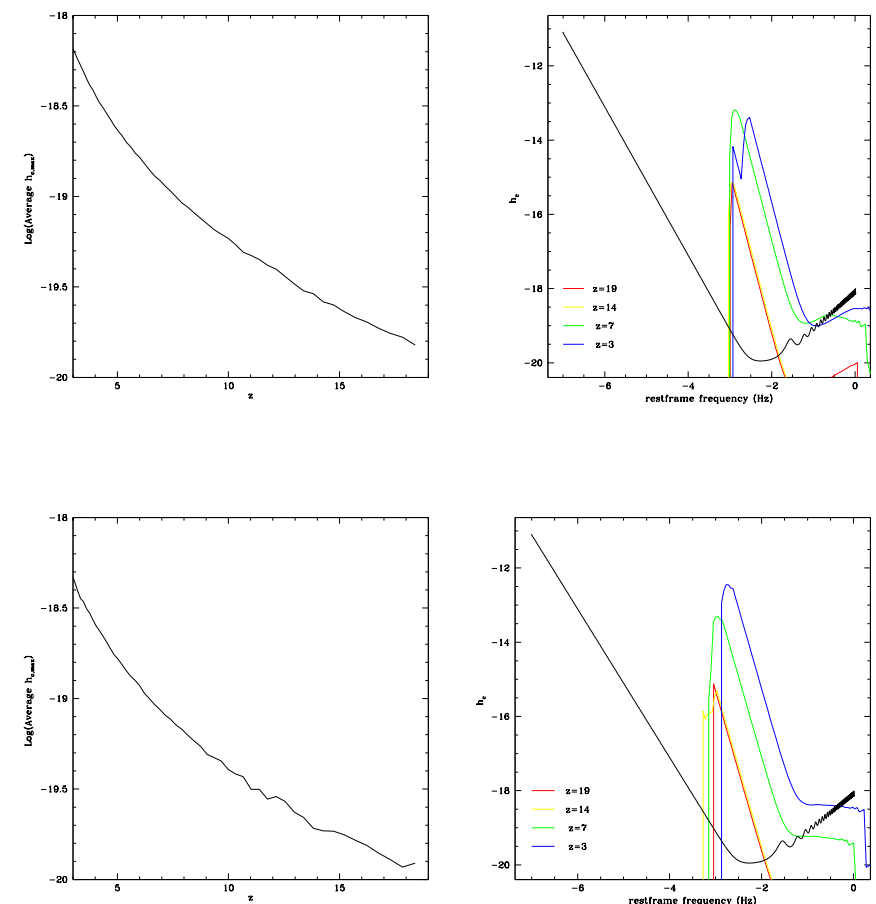

FIGURE 4. 4a-up left- shows the average observed characteristic gravitational strain amplitude as a function of observed redshift. Distance is measured from the center of the primary. Gravitational strain amplitude increases toward smaller redshifts as IMBHs sink into gravitational potential of the primary. This increase is rapid since the ratio of IMBHs binaries increases too. 4b-right- LISA sensitivity curve (black) and the total gravitational strain amplitude at different redshifts. The increase in mass of merging black holes with redshift results in increasing total gravitational strain amplitude and shifting toward higher observed frequencies. $4 \mathrm{c}$ and $4 \mathrm{~d}$ on the bottom - gas accretion added.

\section{ACKNOWLEDGMENTS}

We acknowledge the support of the Center for Gravitational Wave Physics funded by the NSF under cooperative agreement PHY 01-14375, NSF grants PHY 98-00973.

\section{REFERENCES}

Abel et al. 2002. Abel, T., Bryan, G., \& Norman, M., 2002, Sci, 295, 93A

Cornish \& Levin 2002. Cornish, N. J., \& Levin, J., 2002, gr-qc/0207016

Heger et al. 2003. Heger et al., 2003, 2003, ApJ, 591, 288H

Holley-Bockelmann et al. 2001. Holley-Bockelmann, K., \& Mihos, J.C., Sigurdsson, S., Hernquist, L., 2001, ApJ, 549, 862

Islam et al. 2003. Islam, R. R., Taylor, J. E., Silk, J., \& 2003, MNRAS, 340, 647I

Madau \& Rees 2001. Madau, P., Rees M. J., 2001, ApJ, 551L, 27M

Thorne 1995. Thorne, K., 1995, NYASA, 759, 127

Vecchio et al. 2004. Vecchio, A., Wickham, E. D., Stevens, I. R., \& 2004, SPIE, 5500, 183V

Vine \& Sigurdsson 1998. Vine, S., Sigurdsson, S., \& 1998, MNRAS, 295, 475

Volonteri et al. 2003. Volonteri, M., Haardt, F., \& Madau, P., 2003, ApJ, 582, 559

Wise \& Abel 2005. Wise, J., H., Abel, T., 2005, ApJ, 629, 615W 